\title{
QoS based Adaptive Admission Control Algorithm for Wireless Multimedia Networks
}

\author{
Vijayalakshmi M \\ Associate professor in the department of computer \\ Science and Engg, BVBCET, Hubli.
}

\author{
Linganagouda Kulkarni \\ Director \\ JPNES, Mahabubnagar, AP.
}

\begin{abstract}
In wireless multimedia networks, the process of offering Quality of Service (QoS) guaranteed multimedia services becomes more challenging owing to the limited bandwidth, dynamic environment and mobility. This problem can be overcome by a call admission control mechanism that restricts the network access based on resource availability. In this paper QoS based Adaptive admission control algorithm for wireless multimedia networks is proposed. Initially the calls entering the network cell are categorized as real time handoff calls, non-real time handoff calls and new calls which are handled in the similar progression. In common, any call category entering the cell undergoes bandwidth test and when the bandwidth value is satisfied, the call is admitted. Otherwise cell performs different cell resizing technique for respective call categories. The proposed work is implemented in Network simulator (NS-2). It is shown that the proposed admission control algorithm achieves better bandwidth utilization, fairness with reduced call dropping rate, when compared with the existing technique. A mobile cellular architecture based on WCDMA is considered for implementation of this technique.
\end{abstract}

\section{Conclusion}

The proposed methodology prevents call rejection thereby avoid data losses.

\section{Keywords}

Wireless Multimedia Networks, Quality of Service (QoS), Adaptive Admission Control.

\section{INTRODUCTION}

\subsection{Wireless Multimedia Networks}

The real-time multimedia services such as video streaming, video conferencing and Internet Protocol television (IPTV) are experiencing emerging needs in the latest era. The enhanced multimedia services and facilities are offered by mobile devices such as smart phones, personal digital assistants (PDA) and laptops [1]. The people has right to access the multimedia services any time at any place utilizing diverse access networks and computing devices which are entirely facilitated by the wireless multimedia networks.

Wireless networking possesses varied applications commencing from voice communication performed through the cellular phones till the high performance multimedia networking [2]. The multimedia data is extensively utilized in the inspection areas, homeland security, transportation, distance learning, health care and so on. [1]

The key problems in wireless multimedia networking are as follows. [2]

1) Network functioning with restricted energy resources.
2) The necessity of upholding quality of service (QoS) such as throughput, delay, bit error rate etc on timevarying channels.

3) Functioning within a heterogeneous scenario.

\subsection{Quality of Service (QoS) in Wireless Multimedia Networks}

The process of offering a sort of assurance regarding the service level issued to an application is referred to as Quality of service (QoS). The QoS parameters have various forms which are employed accordingly as per the requirements of an application [3]. When compared to static wired networks, offering QoS in wireless mobile networks is difficult. The QoS in wireless networks are accounted at two levels namely application and connection level [4].

- Application-level QoS: This is associated with the quality observed at the user end which is generally considered in packet-switched networks. The parameters used to illustrate the application-level QoS include delay/delay jitter, error/loss, throughput and so on.

- Connection-level QoS: This is associated with connection establishment and organization. It determines the connectivity and continuity of service in the wireless network. The parameters used to illustrate the connection level QoS are new-call blocking probability and handoff-dropping probability.

Some of the QoS requirements of multimedia network are as follows [5]:

- End-to-end Latency: The latency occurrence across a link is termed as end-to-end audio latency. It can also be defined as the time difference of packets forwarded at one end of the link and reception at other end of the link.

- Delay: The time difference between the release of initial bit of a data block by the transmitting endsystem and reception by the end-system is referred as network transmit delay.

- Jitter: The discrepancies of delay created by the transmission equipment are termed as jitter in transmission technology.

- Data Rate: The rate at which the multimedia data's are encoded prior to transmission is termed as data rate.

- Required Bandwidth: The requisite data transmission rate measured in bits per second refers to the required bandwidth. This takes raw data and overhead into account. 
- Loss Rate: The number of bits lost among two points in telecommunication following transmission is referred to as bit loss rate.

- Error Rate: The occurrence of the invalid bits among two points in telecommunication following the transmission is referred to as error rate.

- Response Time: The time elapsed between the transmission of request and reception of the first response by the user is referred as response time.

\subsection{Challenges of Provisioning QoS in Wireless Networks}

Owing to exclusive features of wireless networks, QoS provisioning in multimedia applications becomes more challenging and some of the challenges are explained below [1]

> Unreliable Physical Channels: Wireless channels are variable and possess limited bandwidth. Due to fading and multi-path effects, wireless channels have high packet loss rate and bit error rate. As the wireless medium is shared by multiple stations, the allocation of bandwidth to single stations will affect the neighboring stations. [1]

$>$ Node Mobility: The mobile devices keep wandering and the wireless networks that are connected to them frequently get shifted. The varying environment in the wireless network can result in unreliable bandwidth and rapidly changing error rates owing to the mobility and interference. [6]

$>$ Routing: The topology of the mobile ad hoc networks keeps changing vigorously due to the mobile nature of mobile devices. The routes existing in the network may disappear or deficit of supporting QoS which necessitates the route changes.

$>$ Resource Constraints: The various forms of QoS such as transmission rate, delay and packet dropping ratio are revealed by high speed multimedia packet based applications. It is very complicated to fulfill the requirements since there are only limited radio resource, the time-changing channel condition and the resource contention between the multiple users. [7]

$>$ Heterogeneity: The end-to-end provision of the QoS is more complex due to the heterogeneity of the mobile devices, access and infrastructure networks.

\subsection{Problem Identification}

The literature has very few works for QoS assured wireless multimedia networks. Also, there is no complete and efficient solution to handle different types of calls in the network.

In [9], Ning Lu et al. have proposed utility-based bandwidth adaptation scheme for multi class traffic in wireless networks. In that, if the call do not meet bandwidth requirements even after bandwidth upgradation and degradation process, then the call is rejected. For real time call cases, this rejection leads to significant data loss.

To avoid the above-discussed problem, a new adaptive admission control algorithm is proposed in this paper, which assures QoS in wireless multimedia networks.

\section{RELATED WORK}

Jia Tang et al. [8] have proposed a cross-layer resource allocation scheme for relay networks. Their cross layer scheme characterizes the delay QoS constraints by incorporating the information theory with the effectual capacity. They developed the associated resource allocation algorithm over both amplifyand forward and forward relay networks. The problem existing in this paper is that it does not consider optimal relay protocol which can reduce the network performance.

Ning $L \boldsymbol{u}$ et al. [9] have proposed an integrated utility-fair bandwidth adaptation scheme for multi-class traffic in wireless networks. They assigned a utility function to every call as per adaptive nature. The allocated bandwidth is either upgraded or degraded based on the network load so that network calls acquires the fair utility. They incorporated the adequate call admission control and bandwidth reservation technique which guarantees the QoS to the new and handoff calls.

Dusit Niyato et al. [10] have presented a novel integrated analytical framework for analyzing the quality-of-service (QoS) performance measures in a wireless mobile multimedia network. With guard channel-based call admission control and fairness based adaptive channel allocation at the network layer, ARQ-based error control at the link layer, and adaptive modulation at the physical layer, they analyzed the QoS performances for real-time, non-real-time, and best-effort traffic. Their framework includes an adaptive traffic shaping mechanism that takes allocated channels and channel quality into account for controlling the packet arrival rate.

Wen-Hsing Kuo et al. [11] have proposed utility-based resource allocation for soft QoS traffic in infrastructure-based wireless networks. By the term soft QoS traffic they mean the traffic that demands certain amount of bandwidth for normal operation but permits some flexibility when the given bandwidth is near to the preferred value. They aimed at maximizing the total utility of all soft QoS flows without going through a wireless bidding process. Then, they have proposed a sub-optimal, polynomial time solution based on the developed theorems.

Olabisi E. Falowo et al. [12] have proposed adaptive bandwidth management and Joint Call Admission Control scheme (JCAC) scheme for enhancing system utilization and connection-level QoS in heterogeneous cellular networks. Their scheme enhances average system utilization by adapting the bandwidth of calls based on current traffic condition and by uniformly distribute traffic load among the available Radio Access Technologies (RATs). Also it guarantees the QoS requirements of all accepted call and reduces both new call blocking probability and handoff call dropping probability in the heterogeneous wireless networks.

\section{QoS BASED ADAPTIVE ADMISSION CONTROL}

\subsection{Overview}

In this paper, a QoS based novel call admission control algorithm for wireless multimedia networks is proposed. Initially, the calls entering the network are classified as real time handoff calls $(\mathrm{RH})$, non-real time handoff calls $(\mathrm{NH})$ and new calls (NC). These calls are handled such that the real time handoff calls are given first priority followed by the non-real time calls and finally the new calls. When any call enters the cell, initially its required bandwidth is compared with the available bandwidth of the cell. If the bandwidth value matches, then the corresponding calls are admitted into the cell. While handling $\mathrm{RH}$ calls, if the required bandwidth exceeds the 
available bandwidth, then the cell requests the neighboring cells and chooses a cell with higher available bandwidth which is marked as ready cell. The requested cell reduces its cell size to expel the RH call, and upgrades its bandwidth left by the RH call. The ready cell increases its cell size and accommodates the $\mathrm{RH}$ call. While handling $\mathrm{NH}$ calls, if the bandwidth value exceeds, the cell degrades bandwidth of the ongoing call and attempts to allocate $\mathrm{NH}$ call. Otherwise, $\mathrm{NH}$ call waits until it gets the adequate bandwidth. While handling NC calls, the calls are initially classified into real time and non-real time calls. Based on available bandwidth of the cell, the minimum requirement of the real time NCs are allocated first. If there is no adequate bandwidth for non-real time NCs, then it will be placed in a queue until getting minimum bandwidth requirement.

\subsection{Estimation of Metrics}

\subsubsection{Estimation of Available Bandwidth of a Cell}

Let $\mathrm{Z}$ represent the total capacity of the cell.

$P_{i}$ represents the number of $i$ calls entering the cell

$\mathrm{Q}_{\mathrm{i}}$ represents the number of $\mathrm{i}$ handoff calls that exists in the cell during call request.

The total bandwidth allocated $\left(\mathrm{BW}_{\mathrm{i}, \mathrm{aj}}\right)$ for $\mathrm{i}$ calls entering the cell is denoted as $\mathrm{BW}_{\mathrm{Nwi}}$ which is computed using Eq. 1

$$
\mathrm{BW}_{\mathrm{Nwi}}=\sum_{j=1}^{P_{i}} B W_{i, a j}
$$

$\mathrm{BW}_{\mathrm{i}, \mathrm{aj}}$ for $\mathrm{i}$ handoff calls is denoted as $\mathrm{BW}_{\mathrm{Hdi}}$ which is computed using Eq. 2

$$
\mathrm{BW}_{\mathrm{Hdi}}=\sum_{j=1}^{Q_{i}} B W_{i, a_{j}}
$$

Hence the available bandwidth (AB) is given by Eq: 3

$$
\mathrm{AB}=\mathrm{Z}-\sum_{i=1}^{n}\left(B W_{N w_{i}}+B W_{H d_{i}}\right)
$$

\subsubsection{Estimation of Utility Function}

The utility function corresponds to the level of satisfaction of a user. It naturally reflects the QoS needs of the user and adaptability of the application. It is represented as curve that maps the bandwidth acquired by an application to the performance realized by the user [9].

\subsubsection{Utility function for Real time traffic}

The utility function for modeling the real time multimedia traffic is as shown in Eq: 4

$$
\mathrm{U}(\mathrm{BW})=1-\mathrm{e}^{-\frac{s_{1} B W^{2}}{s_{2}+B W}}
$$

Where $s_{1}$ and $s_{2}$ are variables that estimates the shape of the utility function and guarantees the following scenario.
If maximum bandwidth (BWmax) is received

Then

End if

$$
\mathrm{U} \approx 1
$$

\subsubsection{Utility Function of Non-Real Time Traffic}

The utility function for modeling the non-real time multimedia traffic is as shown in .Eq: 5

$$
\mathrm{U}(\mathrm{BW})=1-\mathrm{e}^{-\frac{s B W}{B W_{\max }}}
$$

where $\mathrm{s}$ is the variable that determines the shape of the utility function and guarantees the following scenario.

$$
\begin{aligned}
& \text { If } \mathrm{BW}_{\text {max }} \text { is received } \\
& \text { Then } \\
& \text { End if }
\end{aligned}
$$

\subsubsection{Bandwidth Adaptation Methodology}

The bandwidth adaptation methodology offers QoS guarantees in wireless networks. The two procedures involved in bandwidth adaptation are bandwidth upgradation and degradation process which is described in section 3.2.3.1 and 3.2.3.2.

\subsubsection{Bandwidth Upgradation}

Whenever a call within the cell ceases or a call departs from the existing cell, the bandwidth upgradation process is activated.

Let us consider a saturated cell with $\mathrm{n}$ outgoing calls

When call within the cell ceases or departs to another cell, the $\mathrm{n}$ outgoing calls might not have received their maximum bandwidth. For upgrading these ongoing calls, the released bandwidth can be used.

Thus the upgradable utility function $\mathrm{U}_{\mathrm{i}}\left(\mathrm{BW}_{\mathrm{i}}\right)$ of the ith outgoing call is given as

$$
\mathrm{U}_{\mathrm{i}}\left(\mathrm{BW}_{\mathrm{i}}\right)=\mathrm{U}_{\mathrm{i}}\left(\delta_{i}+\mathrm{BW}_{\mathrm{i}}\right) \quad 0 \leq \mathrm{BW}_{\mathrm{i}} \leq \rho_{i}
$$

Here $\rho_{i}=\mathrm{BW}_{\text {maxi }}-\delta_{i}$ where $\mathrm{i}=1,2 \ldots \ldots \mathrm{n}$

$\delta_{i}$ represent the currently allocated bandwidth

The main aim of bandwidth upgradation is to estimate the profile of bandwidth upgradation $\left(\mathrm{BW}_{\mathrm{i}}\right)$ for $\mathrm{n}$ outgoing calls such that equal utility value can be obtained by every call.

$$
\begin{gathered}
\text { i.e. } 1 \leq \forall i, \mathrm{j} \leq \mathrm{n}, \\
\mathrm{U}_{\mathrm{i}}\left(\delta_{i}+\mathrm{BW}_{\mathrm{i}}\right)=\mathrm{U}_{\mathrm{j}}\left(\delta_{j}+\mathrm{BW}_{\mathrm{j}}\right)
\end{gathered}
$$

\subsubsection{Bandwidth Degradation}

Whenever a new call is generated within the cell or a handoff call enters into the cell, the bandwidth degradation process is activated. i.e. the bandwidth of ongoing calls is degraded to smaller values to accommodate the new or handoff calls.

Thus the degradable utility function $\mathrm{Ui}\left(\mathrm{BW}_{\mathrm{i}}\right)$ of the ith ongoing call is given as

$$
\mathrm{U}_{\mathrm{i}}\left(\mathrm{BW}_{\mathrm{i}}\right)=\mathrm{U}_{\mathrm{i}}\left(\delta_{i}-\mathrm{BW}_{\mathrm{i}}\right), 0 \leq \mathrm{BW}_{\mathrm{i}} \leq \delta_{i}
$$

The utility function of the new or handoff calls is denoted as $\mathrm{U}_{\mathrm{n}+1}\left(\mathrm{BW}_{\mathrm{n}+1}\right)$. 
Thus the main aim of bandwidth degradation process is to estimate the bandwidth allocation $\mathrm{BW}_{\mathrm{n}+1}$ for the new or handoff call such that the utility is distributed equally among all ongoing calls and new or handoff call.

$$
\begin{aligned}
& \text { i.e. } 1 \leq \forall i, \mathrm{j} \leq \mathrm{n} \\
& \mathrm{U}_{\mathrm{i}}\left(\delta_{i}-\mathrm{BW}_{\mathrm{i}}\right)=\mathrm{U}_{\mathrm{j}}\left(\delta_{i}-\mathrm{BW}_{\mathrm{j}}\right)=\mathrm{U}_{\mathrm{n}+1}\left(\mathrm{BW}_{\mathrm{n}+1}\right) .
\end{aligned}
$$

\subsection{Classification of Handoff Calls}

The Handoff calls entering into the network are categorized into following three classes.

Class 1: Real time handoff Calls (RH)

Class 2: Non-real time handoff calls $(\mathrm{NH})$

\section{Class 3: New Calls (NC)}

The above classes of handoff calls are handled according to the priority levels.

$$
\text { i.e. } \mathrm{RH}>\mathrm{NH}>\mathrm{NC}
$$

This reveals that when the calls enter the network, RH calls are given higher priority. Then $\mathrm{NH}$ calls are given the second higher priority and the NC calls are given lower priority.

The handoff call management technique is explained in section 3.4

\subsection{Call Management Technique}

The call management technique carries three sections which are as follows

1) Handling real time handoff calls,

2) Handling non-real time handoff calls,

3) Handling new calls

\subsubsection{Algorithm for Handling Real Time Handoff Calls}

When the real time handoff $(\mathrm{RH})$ calls enter into a new cell, they are handled as per the following steps.

1) Initially the required bandwidth of $\mathrm{RH}$ call $\left(\mathrm{BW}_{\text {req }}\right)$ is compared with available bandwidth of the cell $(\mathrm{AB}(\mathrm{C}))$.

1.1 If $\left(\mathrm{BW}_{\text {reqr }}\right) \leq \mathrm{AB}(\mathrm{C})$ then

1.1.1 $\mathrm{C}$ admits the RH call.

\subsection{Else}

1.2.1 $\mathrm{C}$ degrades the bandwidth of ongoing calls and attempts to allocate the $\mathrm{RH}$ calls.

$$
\text { 1.2.2 If }\left(\mathrm{BW}_{\text {reqr }}\right) \leq \mathrm{AB}(\mathrm{C}) \text { then }
$$

1.2.3 $\mathrm{C}$ admits the $\mathrm{RH}$ call.

$$
1.3 \text { Else }
$$$$
\text { 1.3.1 Goto } 2
$$

\subsection{End if}

\subsection{End if}

2) $\mathrm{C}$ sends bandwidth query message (BW_QUERY) message to all neighboring cells $\left(\mathrm{C}_{\text {neigh }}\right)$.

$$
\mathrm{C} \stackrel{B W_{-} Q U E R Y}{\longrightarrow} \mathrm{C}_{\text {neigh }}
$$

3) Upon receiving BW_QUERY messages, each cell computes its available bandwidth (AB) (Shown in section 3.2.1) and sends it to the requested cell $\left(\mathrm{C}_{\text {req }}\right)$.

$$
C \stackrel{A B}{\longleftarrow} C_{\text {neigh }}
$$

4) The requested cell selects the cell that contains higher $\mathrm{AB}$ and the chosen cell is termed as ready cell $\mathrm{C}_{\text {ready }}$
5) The requested cell then forwards acknowledgment (ACK) message to the ready cell.

$$
\mathrm{C}_{\text {req }} \stackrel{A C K}{\longrightarrow} \mathrm{C}_{\text {ready }}
$$

6) $\mathrm{C}_{\text {req }}$ reduces its cell size to expel the $\mathrm{RH}$ call, and upgrades its bandwidth (shown in section 3.2.3a) which is left by the RH call.

7) Upon receiving ACK message, $C_{\text {ready }}$ increases its cell size and accommodates the RH call.

For example consider a cell structure in Figure 1. There exist 12 cells in the network. Figure 1.a takes two adjacent cells such as cell 3 and cell 4 into consideration. Here, the cell 3 represents the requested cell and cell 4 represents the ready cell. Figure 1.b and Figure 1.c collectively shows the real time handoff management technique. Figure 1.b shows that a RH call arrives at node 5 in cell 3 . As the bandwidth value is not satisfied, cell 3 reduces its size to expel the node 5 to the neighboring cell 4 which has higher available bandwidth and upgrades its bandwidth left by the RH call. Figure 1.c shows that the cell 4 increases in its size to accommodate the node 5 .

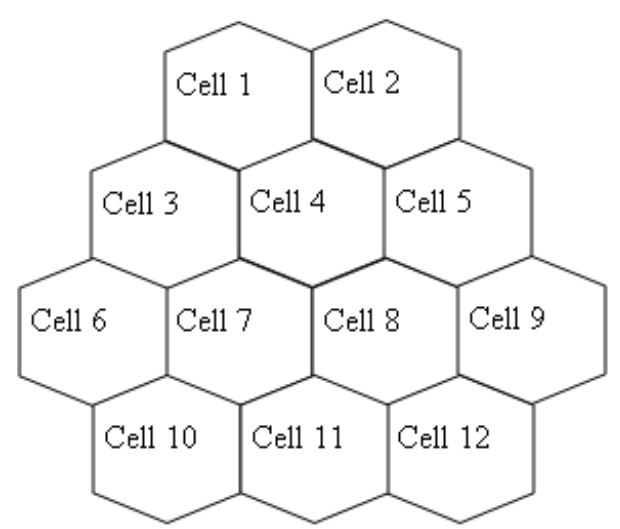

Fig 1 Cell structure
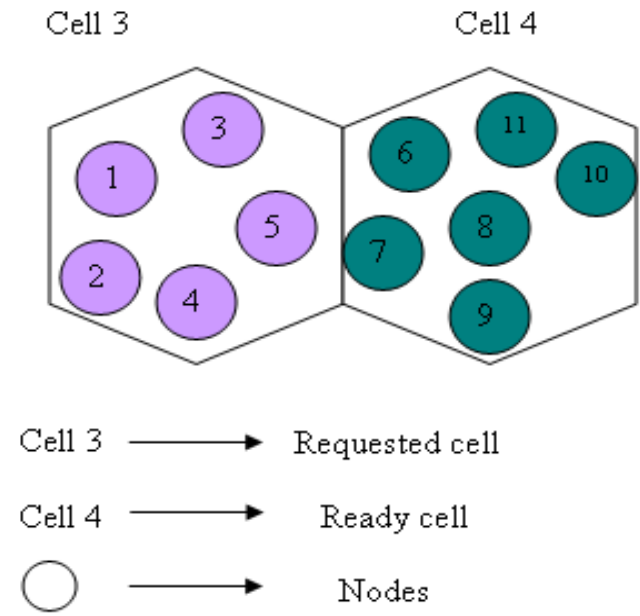

Fig 1. a Example of a reque sted and ready cell 


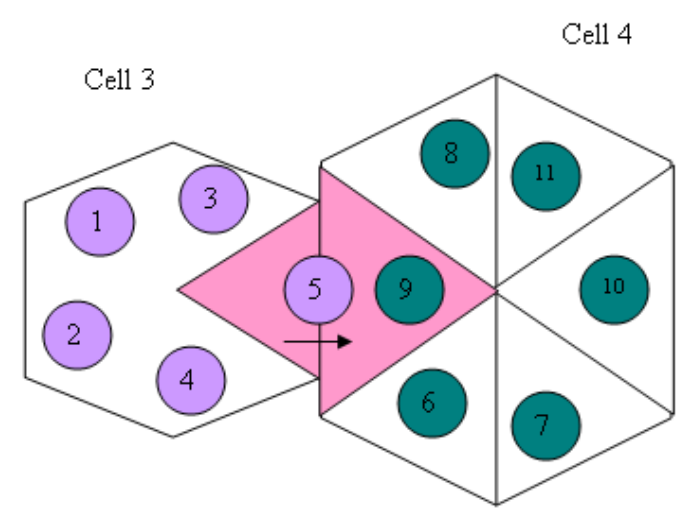

Fig 1.b Reduction in cell 3 size to expel the $\mathrm{RH}$ call

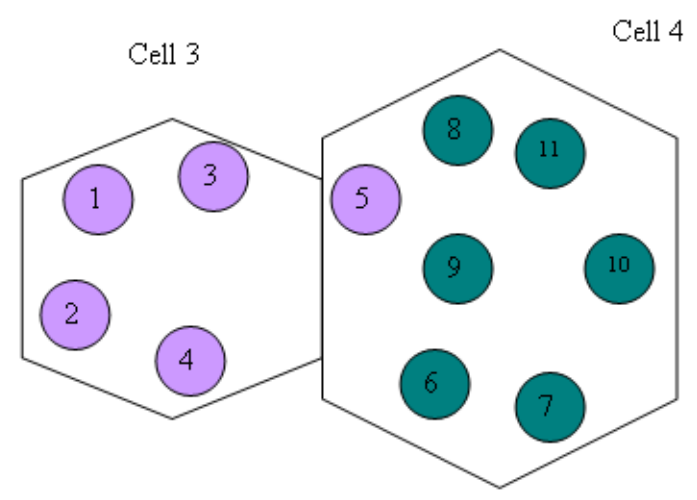

Fig 1.c Increase in size of cell 4 and accommodation of $\mathrm{RH}$ call.

\subsubsection{Algorithm for Handling Non-Real Time Handoff Calls}

When the non-real time handoff $(\mathrm{NH})$ calls enter into a new cell, they are handled as per the following steps.

1) Initially the required bandwidth of $\mathrm{NH}$ call $\left(\mathrm{BW}_{\text {reqn }}\right)$ is compared with available bandwidth of the cell $(\mathrm{AB}(\mathrm{C}))$.

If $\left(\mathrm{BW}_{\text {reqn }}\right) \leq \mathrm{AB}(\mathrm{C})$

Then

$$
\mathrm{C} \text { admits the } \mathrm{NH} \text { call. }
$$

Else if $\left(\mathrm{BW}_{\text {reqn }}\right)>\mathrm{AB}(\mathrm{C})$

$\mathrm{C}$ degrades the bandwidth of the ongoing call and attempts to allocate $\mathrm{NH}$ call.

\section{Else}

$\mathrm{NH}$ call waits until it gets the adequate bandwidth.

End if

\subsubsection{Algorithm for Handling New Calls}

The steps involved in handling new calls are as follows.

1) When a new call (NC) arrives at a cell, initially it is classified into real time and non-real time calls.
2) As the real time calls are given higher priority, based on available bandwidth of the cell, the minimum requirement of the real time NCs are allocated first.

3) If there is no adequate bandwidth for non-real time NCs,

Then

Non-real time NCs are placed in a queue until getting minimum bandwidth requirement.

End if

\section{SIMULATION RESULTS \\ 4.1. Simulation Setup}

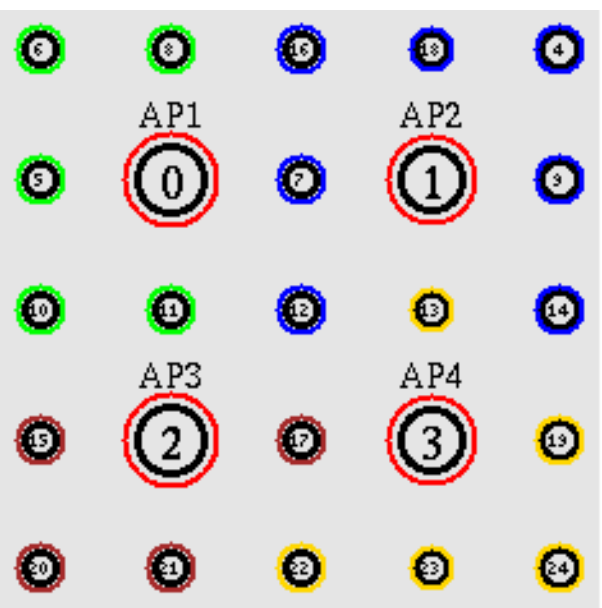

Figure 2: Simulation Topology

The proposed QoS Based Adaptive Admission Control (QAAC) algorithm is simulated in NS-2 [14], which is a general-purpose simulation tool that provides discrete event simulation of user defined networks. In the simulation, in a 100 meter $x 100$ meter region, 4 cells are arranged with $\mathrm{APs} \mathrm{AP} 1, \mathrm{AP} 2, \mathrm{AP} 3$ and AP4. Initially AP1 has 5 clients, AP2 has 7 clients, AP3 has 4 clients and AP4 has 5 clients. The simulation topology is given in Figure 2. The simulation is conducted for 50 seconds of simulation time. All nodes have the same transmission range of 250 meters. The simulation parameters are given in table 1 . For each user in the cell, both UDP and Video traffic are tested.

\begin{tabular}{|l|l|}
\hline Area Size & $100 \mathrm{X} 100$ \\
\hline Number of Cells & 4 \\
\hline Users Per Cell & $5,7,4,5$ \\
\hline Slot Duration & $2 \mathrm{msec}$ \\
\hline Radio Range & $250 \mathrm{~m}$ \\
\hline Simulation Time & $50 \mathrm{sec}$ \\
\hline Routing Protocol & AODV \\
\hline Traffic Source & CBR and Video \\
\hline Packet Size & 512 bytes \\
\hline MSDU & 2132 \\
\hline $\begin{array}{l}\text { Initial Load of } \\
\text { users }\end{array}$ & $250 \mathrm{~kb}$ \\
\hline No. of Users & 1 to 5 \\
\hline Transmit Power & $0.660 \mathrm{w}$ \\
\hline Receiving Power & $0.395 \mathrm{w}$ \\
\hline IdlePower & $0.035 \mathrm{w}$ \\
\hline Initial Energy & $5.1 \mathrm{~J}$ \\
\hline
\end{tabular}




\subsection{Performance Metrics}

The proposed QAAC algorithm is compared with utility-fair bandwidth adaptation scheme (UBA) [9] for multi-class traffic. The performance metrics are:

Fairness: It is the ratio of bandwidth received into total available bandwidth for a traffic flow.

Bandwidth: It is the bandwidth received successfully, measured in $\mathrm{Mb} / \mathrm{s}$.

Average Energy: It is the average energy consumption of all nodes in sending, receiving and forward operations.

The load of the users in the cells AP1, AP2 and AP4 are gradually increased in steps of $50 \mathrm{~kb}$. The results of applying the QAAC algorithm are presented in Figure 3 and the topology after cell resizing is given in Figure 4.

The above metrics are measured for AP1, AP2 and AP4 with UDP and Video traffic scenarios. In all the experiments, the number of users in each cell is varied.

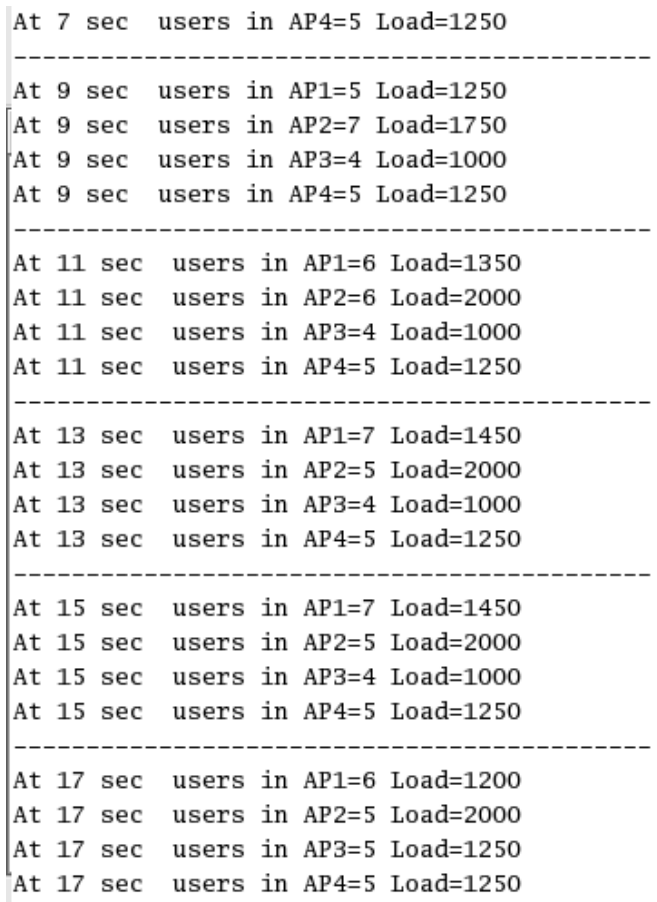

Figure 3: Results of Cell Resizing



Figure 4: Topology of Resized Cells
Case-1(UDP)

For AP1

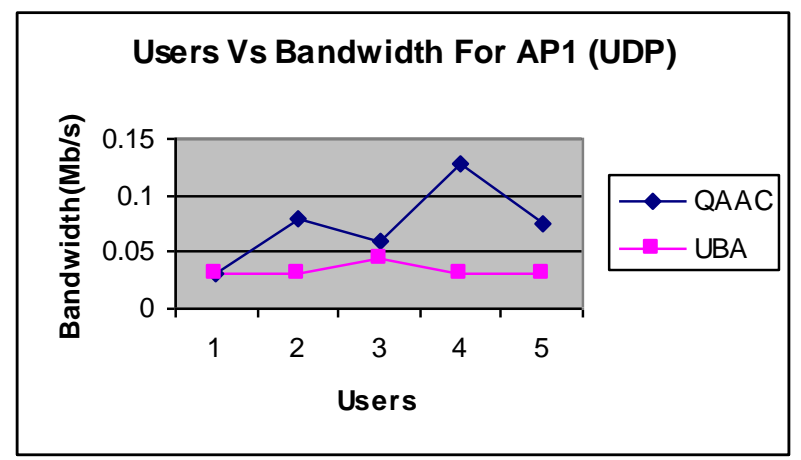

Figure 5: Users Vs Bandwidth

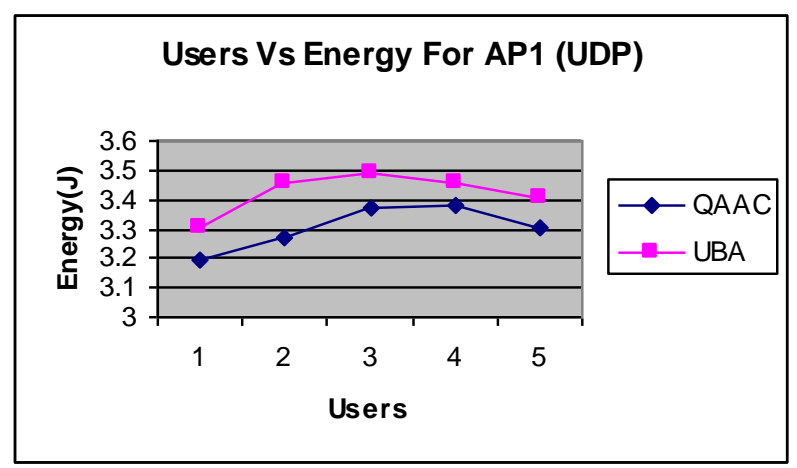

Figure 6: Users Vs Energy

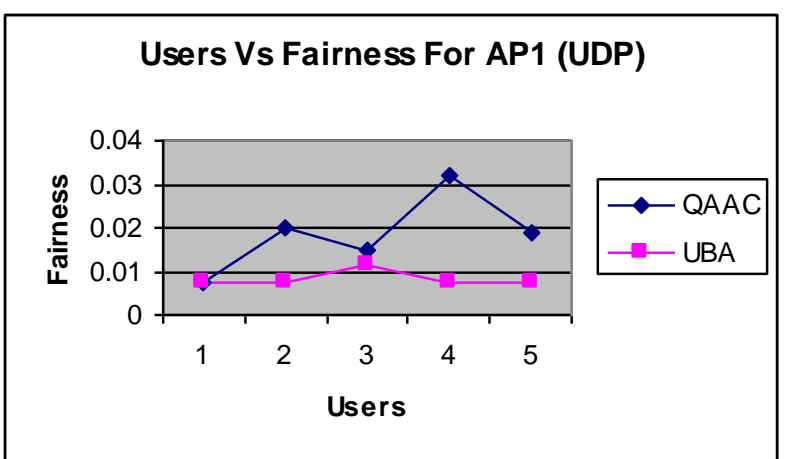

Figure 7: Users Vs Fairness

For AP2

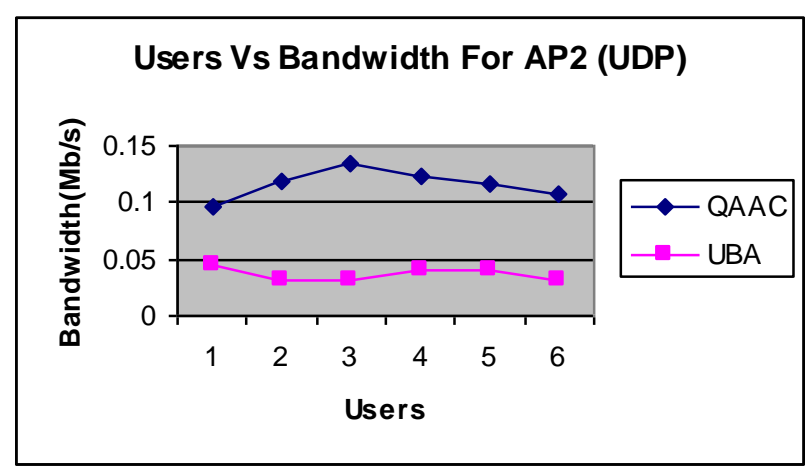

Figure 8: Users Vs Bandwidth

The performance results are presented in the next section. 


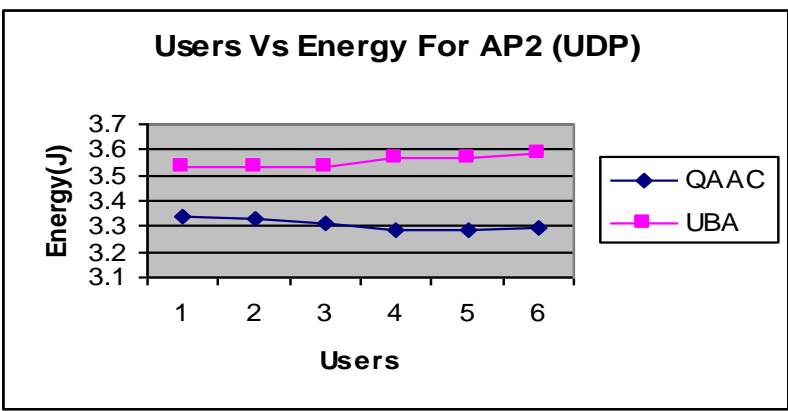

Figure 9: Users Vs Energy

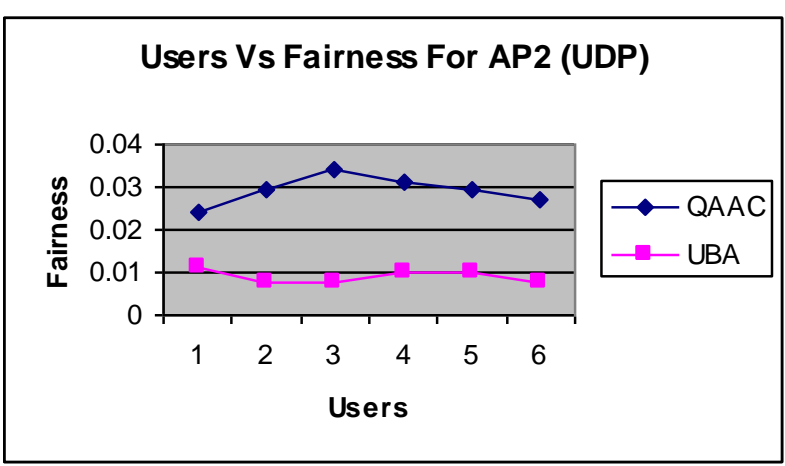

Figure 10: Users Vs Fairness

\section{For AP4}

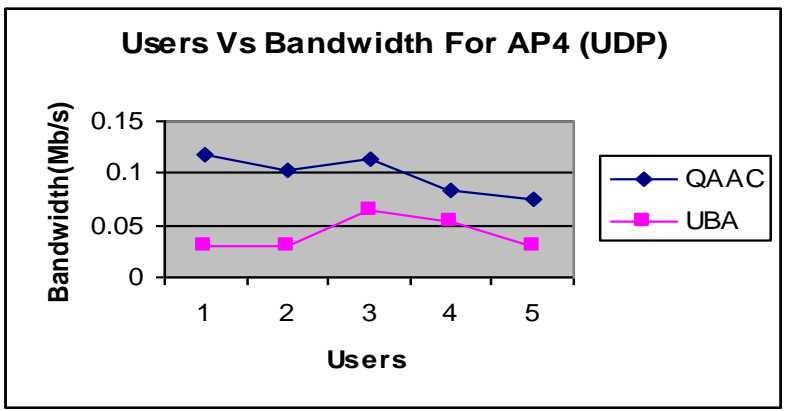

Figure 11: Users Vs Bandwidth



Figure 12: Users Vs Energy

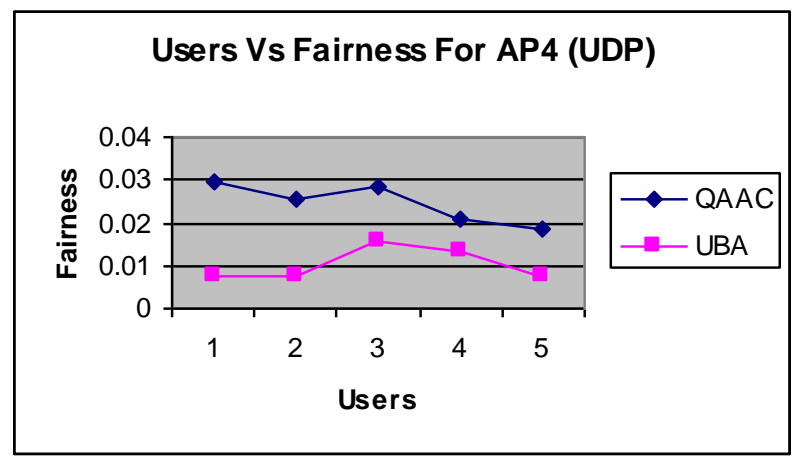

Figure 13: Users Vs Fairness

The figure 5, 8 and 11 shows the received bandwidth for AP1, AP2 and AP4, respectively, while using non real-time traffic flows. It shows that, when the number of users is increased from 1 to 5, QAAC attains increased bandwidth when compared to the UBA scheme.

The figure 7, 10 and 13 shows the fairness value for AP1, AP2 and AP4, respectively, while using non real-time traffic flows. It shows that, when the number of users is increased from 1 to 5 , QAAC attains increased fairness when compared to the UBA scheme.

The figure 6, 9 and 12 shows energy consumed for AP1, AP2 and AP4, respectively, while using non real-time traffic flows. It shows that, when the number of users is increased from 1 to 5 , QAAC has reduced energy consumption when compared to the UBA scheme.

Case-2 (Video)

For AP1

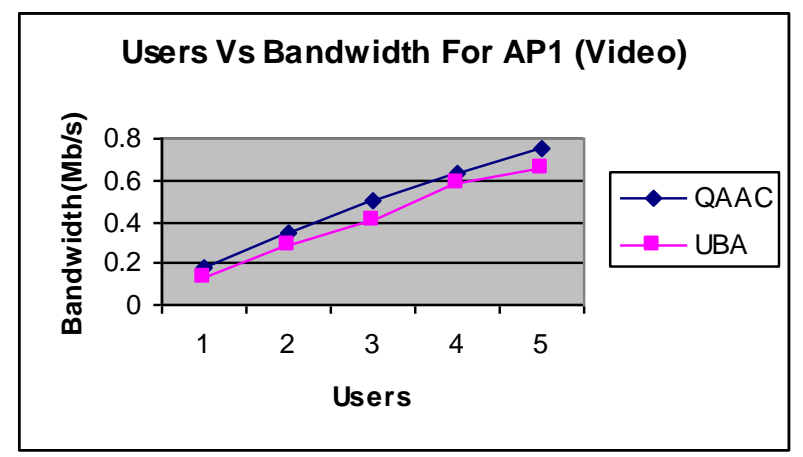

Figure 14: Users Vs Bandwidth

Users Vs Energy For AP1 (Video)

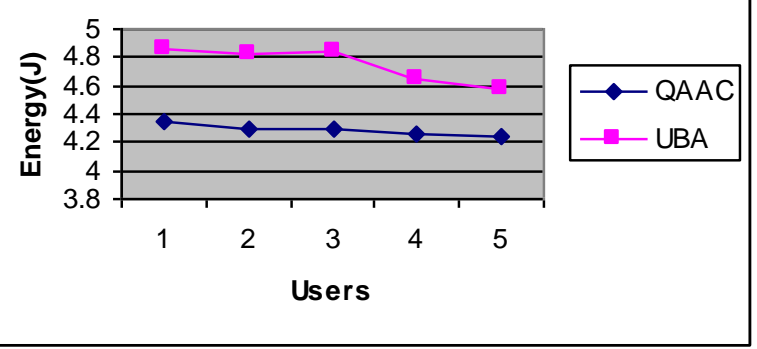

Figure 15: Users Vs Energy 


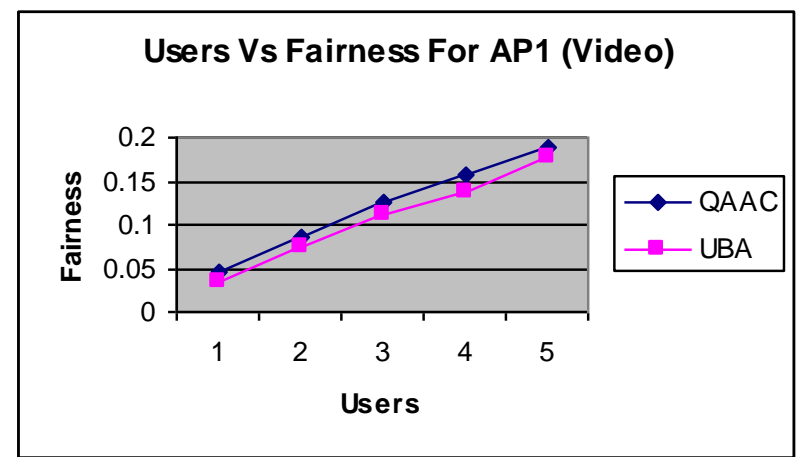

Figure 16: Users Vs Fairness

\section{For AP2}

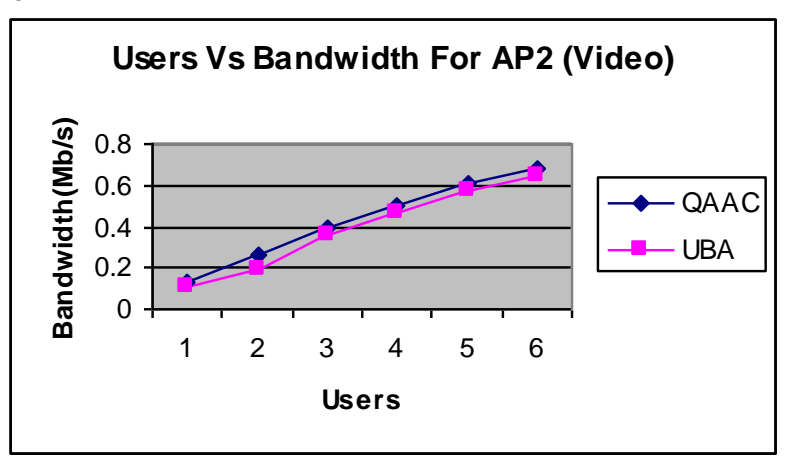

Figure 17: Users Vs Bandwidth

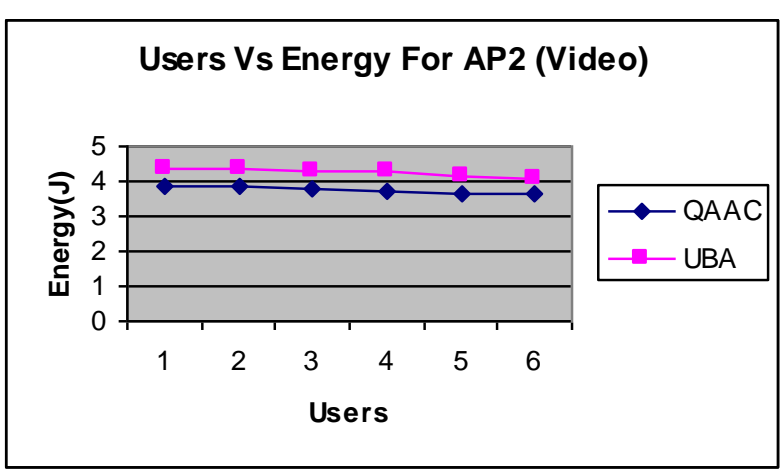

Figure 18: Users Vs Energy



Figure 19: Users Vs Fairness

For AP4

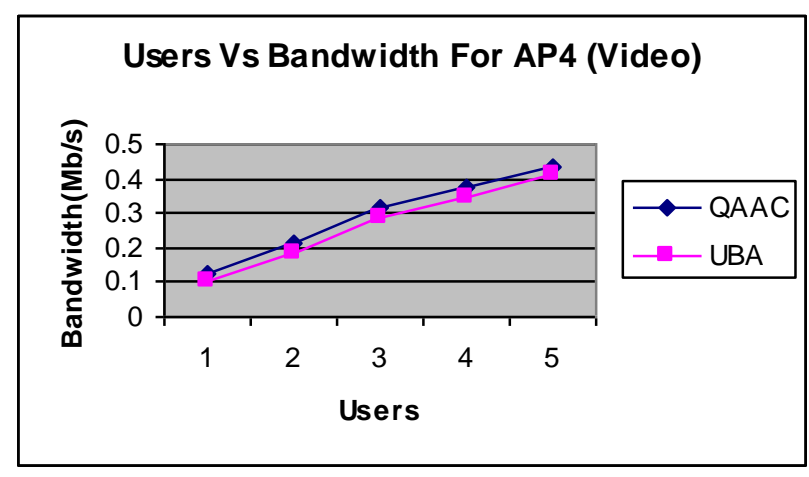

Figure 20: Users Vs Bandwidth

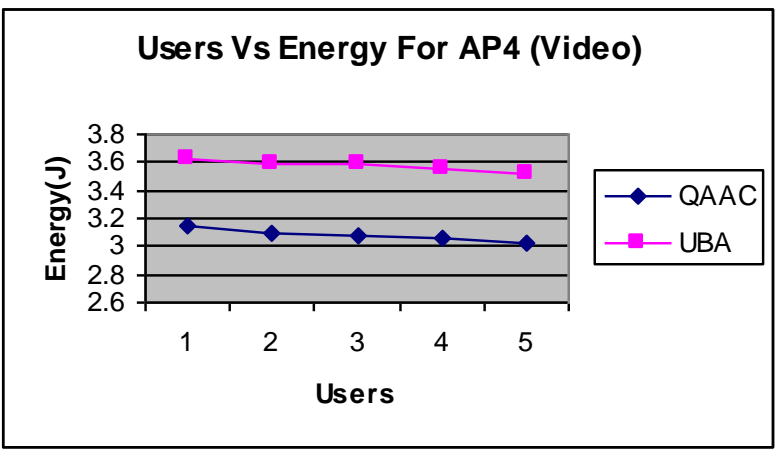

Figure 21: Users Vs Energy

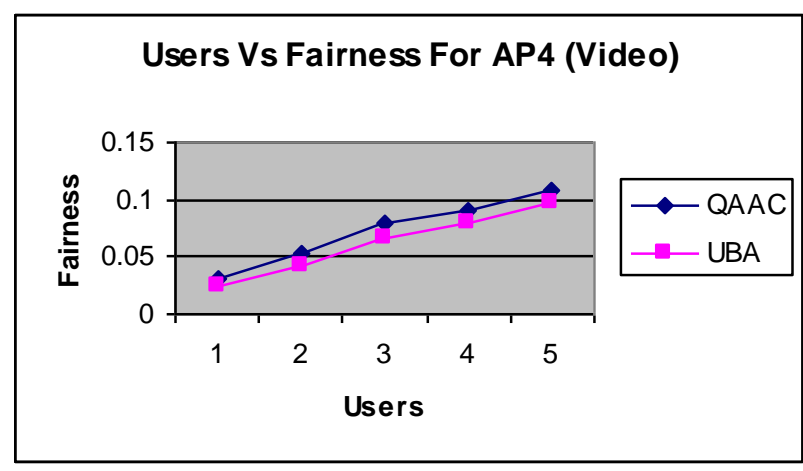

Figure 22: Users Vs Fairness

The figure 14, 17 and 20 shows the received bandwidth for AP1, AP2 and AP4, respectively, while using real-time traffic flows. It shows that, when the number of users is increased from 1 to 5, QAAC attains slightly increased bandwidth when compared to the UBA scheme.

The figure 16, 19 and 22 shows the fairness value for AP1, AP2 and AP4, respectively, while using real-time traffic flows. It shows that, when the number of users is increased from 1 to 5 , QAAC attains slightly increased fairness when compared to the UBA scheme.

The figure 15, 18 and 21 shows energy consumed for AP1, AP2 and AP4, respectively, while using real-time traffic flows. It shows that, when the number of users is increased from 1 to 5 , QAAC has significantly reduced energy consumption when compared to the UBA scheme.

\section{CONCLUSION}

In this paper a QoS based Adaptive admission control algorithm for wireless multimedia networks has been proposed. Initially the calls entering the network cell are categorized as real time 
handoff calls $(\mathrm{RH})$, non-real time handoff $(\mathrm{NH})$ calls and new calls (NC). These calls are handled such that the real time handoff calls are given first priority followed by the non-real time calls and finally the new calls. In common, any call category entering the cell undergoes bandwidth test and when the bandwidth value is satisfied, the call is admitted. Otherwise cell performs different cell resizing technique for corresponding call categories. The proposed work is implemented in Network simulator (NS-2). It has been shown that the proposed call admission control algorithm achieves better bandwidth utilization, fairness with reduced call dropping rate, when compared with the existing technique. A mobile cellular architecture based on WCDMA is considered for implementing this algorithm. Power management and delay aware scheduling are left as potential problems for future work.

\section{REFERENCES}

[1] Hongli Luo and Mei-Ling Shyu, "Quality of service provision in mobile multimedia - a survey", Springer, Human centric Computing and Information Sciences, 2011

[2] Paul J.M. Havinga and Gerard J.M. Smit, "Energy-efficient wireless networking for multimedia applications", Wireless Communications and Mobile Computing (WCMC), pp- 165-184, 2005.

[3] Ash Mohammad Abbas and Øivind Kure, "Quality of Service in mobile ad hoc networks: a survey", Inderscience, International Journal of $\mathrm{Ad} \mathrm{Hoc}$ and Ubiquitous Computing, 2008.

[4] Lei Huang, Sunil Kumar and C.-C. Jay Kuo, "Adaptive Resource Allocation for Multimedia QoS Management in Wireless Networks", IEEE Transactions on Vehicular Technology, 2004.

[5] Yan Chen, Toni Farley and Nong Ye, "QoS Requirements of Network Applications on the Internet" Journal on Information-Knowledge-Systems Management, Volume 4 Issue 1, January 2004.
[6] Bobby Vandalore, Raj Jain, Sonia Fahmy and Sudhir Dixit, "AQuaFWiN: Adaptive QoS Framework for Multimedia in Wireless Networks and its Comparison with other QoS Frameworks", IEEE Conference on Local Computer Networks, (LCN '99), 1999.

[7] G. Indumathi and K. Murugesan, "A Cross-Layer Resource Scheduling with QoS Guarantees Using Adaptive Token Bank Fair Queuing Algorithm in Wireless Networks", Journal of Engineering Science and Technology, pp-260267, 2011.

[8] Jia Tang and Xi Zhang, "Cross-Layer Resource Allocation over Wireless Relay Networks for Quality of Service Provisioning", IEEE Journal on Selected Areas in Communications, 2007.

[9] Ning Lu and John Bigham, "On utility-fair bandwidth adaptation for multi-class traffic QoS provisioning in wireless networks", ELSEVIER, Computer networks, 2007.

[10] Dusit Niyato and Ekram Hossain, “A Novel Analytical Framework for Integrated Cross-Layer Study of Call-Level and Packet-Level QoS in Wireless Mobile Multimedia Networks", IEEE Transactions on Mobile Computing, 2007.

[11] Wen-Hsing Kuo and Wanjiun Liao, "Utility-based Radio Resource Allocation for QoS Traffic in Wireless Networks", IEEE Transactions on Wireless Communications, 2008

[12] Olabisi E. Falowo and H. Anthony Chan, "Adaptive Bandwidth Management and Joint Call Admission Control to Enhance System Utilization and QoS in Heterogeneous Wireless Networks", EURASIP Journal on Wireless Communications and Networking, 2007.

[13] Eduard Garcia, Rafael Vidal and Josep Paradells, "Cooperative Load Balancing in IEEE 802.11 Networks with Cell Breathing", IEEE Symposium on Computers and Communications, (ISCC 2008), 2008.

[14] Network simulator, http://www.isi.edu/nsnam/ns 